\title{
Asociación entre el gen de caveolina 1 (cav1) y el riesgo cardiovascular en adultos
}

\author{
Gustavo Mora García, MD., Ph. D. ${ }^{* 1}$; María Stephany Ruiz Díaz, Méd. Estudiante Ph. D.; \\ Ángelo Alario Bello, Méd. ${ }^{3}$; Doris Gómez Camargo, Bact., MSc., Ph.D.'; Claudio Gómez Alegría, \\ $B Q$, Ph.D. ${ }^{5}$
}

\begin{abstract}
${ }^{1}$ Medicina, Doctorado en Medicina Tropical.
Universidad de Cartagena, Cartagena de Indias, Colombia.

${ }^{2}$ Medicina, Estudiante del Doctorado en Medicina Tropical. Universidad de Cartagena, Cartagena de Indias, Colombia. Internista.

${ }^{3}$ Medicina, Medicina Interna. Universidad de Cartagena, Cartagena de Indias, Colombia.

${ }^{4}$ Bacterióloga, Maestría en Microbiología Clínica, Doctorado en Biología Molecular. Universidad de Cartagena, Cartagena de Indias, Colombia.

${ }^{5}$ Bioquímica, Doctorado en Ciencias Biológicas, Postdoctorado. Universidad Nacional de Colombia, Bogotá, Colombia.
\end{abstract}

Autor de correspondencia:

*Gustavo Mora García

Dirección: Barrio Zaragocilla, Cra. 50 \#24-120. Facultad de Medicina, Universidad de Cartagena.

Celular: +573104236754

Correo: gmorag@unicartagena.edu.co

Posibles conflictos de interés:

Gustavo Mora García y María Stephany Ruiz Díaz fueron financiados por el Departamento Administrativo de Ciencia, Tecnología e Innovación (COLCIENCIAS) mediante la beca para estudiantes doctorales nacionales, convocatorias: 528-2012 y 647-2015 (Res. 2286) respectivamente.

Adicionalmente, el presente trabajo fue financiado

mediante la convocatoria $\mathrm{N}^{\circ} 667-2014$ (código del proyecto 110765741638) por COLCIENCIAS.

Fecha de recepción: 4/12/2017

Fecha de aceptación: 22/01/2018

\section{Resumen}

$\mathrm{L}$ a caveolina 1 es una proteína estructural que interviene en diversos procesos metabólicos. En estudios de asociación genética, el gen que la codifica CAV1 (7q31.2) se ha asociado a resistencia a la insulina, hipertensión, hipertrigliceridemia y c-HDL bajo. Sin embargo, se desconoce si variantes en $C A V 1$ se asocian a obesidad e incremento en el riesgo cardiovascular (RCV).
Objetivo: Analizar la posible asociación entre variantes en CAV1 con el RCV en población del caribe colombiano.

Metodología: Se realizó un estudio de asociación genética con 595 adultos de Cartagena de Indias. Se hizo la genotipificación de los polimorfismos de nucleótido simple (SNPs): rs3779512, rs926198, rs10207569, rs11773845, rs7804372, rs1049337. El RCV fue definido mediante el puntaje derivado del estudio de Framingham. Para estimar la asociación entre las variables de estudio se realizó un análisis de varianza ajustado y fueron construidos árboles de regresión. Se aplicó el ajuste de Bonferroni para pruebas múltiples, donde fuera necesario.

Resultados: La variante rs1049337 se encontró asociada con el incremento del RCV ( $p=0,0001)$. Los sujetos con el genotipo TT en este locus tuvieron un mayor puntaje de RCV, $+4,1$ IC95\% $[0,8-8,0]$ en comparación con CC $(p=0,03)$ y $+4,3$ IC95\% $[0,3-8,4]$ con CT $(p=0,03)$. El árbol mostró que en el grupo TT (rs1049337) presentaba un mayor puntaje si los sujetos además contaban con el genotipo TT para rs3779512.

Conclusiones: Las variantes rs1049337 y rs3779512 se encuentran asociadas a incremento en el puntaje de RCV.

Palabras clave: caveolina 1, enfermedades cardiovasculares, polimorfismo de nucleótido simple, estudios de asociación genética, Colombia, América Latina.

\section{Introducción}

Las enfermedades cardiovasculares (ECV) son un grupo de entidades complejas que incluyen la enfermedad coronaria, la isquemia cardiaca, la enfermedad cerebrovascular, desórdenes vasculares (e.g., hipertensión arterial) y otros trastornos $\operatorname{cardiacos}^{(1)}$. Generalmente, la etiopatogenia es multifactorial con contribución de agentes biológicos (i.e., genéticos, celulares o infecciosos), ambientales y socioeconómicos que conjuntamente incrementan el riesgo de enfermar ${ }^{(2,3)}$.

En América Latina las ECV representan la primera causa de morbilidad y mortalidad en la población adulta ${ }^{(4)}$. Se estima que en la región ocurren más de un millón de muertes anualmente, atribuidas directamente a estas enfermedades y que 
éstas representan alrededor del $42 \%$ de todas las defunciones prematuras ${ }^{(1,4)}$. Además se ha proyectado que para el año 2020 la mortalidad se habrá incrementado en un 60\%, principalmente como consecuencia de la aceleración de las tasas de obesidad y diabetes mellitus tipo 2 (DM2), dos de los más importantes factores de riesgo asociados con las ECV ${ }^{(4,5)}$.

Ante este panorama, el análisis del riesgo cardiovascular (RCV) ha sido ampliamente adoptado como una estrategia útil para el control de la epidemia y la prevención de las ECV ${ }^{(3)}$. El RCV es la probabilidad de desarrollar una ECV dentro de un periodo de tiempo definido, cuya determinación se basa principalmente sobre la concurrencia de factores de riesgo modificables (e.g., hábito de fumar, índice de masa corporal, entre otros) y no modificables (e.g., sexo, etnia, entre otros ${ }^{(6)}$. Con este enfoque se han validado diversos modelos de algoritmos de predicción que emplean combinaciones de estos factores de riesgo para arrojar valores, o puntajes, semicuantitativos ${ }^{(7)}$.

Recientemente algunos algoritmos y ecuaciones han sido enriquecidos con la introducción de biomarcadores y variantes genéticas que tienen como objetivo incrementar la fiabilidad de las predicciones, sobre todo en poblaciones con características heterogéneas, donde la aplicación de métodos generalizados frecuentemente arroja resultados imprecisos ${ }^{(8)}$. En particular, el poder predictivo del riesgo calculado a través de la ecuación de Framingham se ha visto incrementado con la inclusión en el análisis de algunos polimorfismos de nucleótido simple (SNP, por su sigla en inglés Single Nucleotide Polymorphism) en los genes UCP2, ApoE, LPL y ApoA4, al igual que con otros SNPs cercanos al locus 9p2 $1^{(9-12)}$. Es por esto que continúan los esfuerzos por describir las variantes genómicas asociadas con el RCV ${ }^{(13)}$.

Anteriormente nuestro grupo de investigadores analizó la relación del gen de caveolina 1 (CAV1) con la hipertrigliceridemia y el síndrome metabólico ${ }^{(14)}$, por lo que se ha generado la hipótesis de que variantes en este gen podrían estar además asociadas con el RCV. CAV1 está localizado en 7q31.2 y codifica para una proteína integral de membrana de $22 \mathrm{kDa}$, que es indispensable para la formación de estructuras invaginadas en la superficie celular, denominadas cavéolas ${ }^{(15-17)}$. En conjunto, el complejo CAVEOLINA-1/cavéola ha sido identificado como parte de un señalosoma que involucra los receptores de insulina, adiponectina y leptina, cuya actividad depende en parte de la fosforilación del primer dominio de la CAVEOLINA- ${ }^{(18)}$. Por su parte el gen $C A V 1$ se ha asociado con resistencia a la insulina, lipodistrofia, dislipidemia, hipertensión y síndrome metabólico ${ }^{(19-22)}$. En congruencia con estos antecedentes, el objetivo del presente estudio fue analizar la posible asociación entre variantes en $C A V 1$ con el RCV en población adulta.

\section{Materiales y métodos}

Se llevó a cabo un estudio de corte transversal en Cartagena de Indias, una ciudad del caribe colombiano, habitada por cerca de un millón de personas, donde ha sido descrita una acentuada estratificación genética, compuesta por grupos con ancestros europeos (60\%), africanos (30\%) y amerindios $(10 \%)^{(23-26)}$

El estudio incluyó adultos (18-80 años) no emparentados, residentes del área urbana de Cartagena de Indias. Fueron excluidos los sujetos con historia de alteración endocrina primaria o enfermedad de origen genético. Además, fueron descartadas para el análisis las mujeres gestantes y personas con historia de intervenciones quirúrgicas para el tratamiento. Todos los sujetos participaron de forma voluntaria y dieron su consentimiento informado por escrito, según las recomendaciones del Comité de Ética en Investigación de la Universidad de Cartagena.

A los sujetos seleccionados se les midieron las variables antropométricas (i.e., altura, peso, circunferencia abdominal y perímetro de caderas), siguiendo las recomendaciones de la Federación Internacional de Diabetes, y la presión arterial fue medida por el método auscultatorio ${ }^{(27,28)}$. Igualmente, fue recolectada una muestra de sangre venosa bajo condiciones de ayuno no inferior a 8 horas para medir las concentraciones séricas de glucosa, triglicéridos y c-HDL, además, una alícuota de esta muestra fue conservada para análisis genéticos posteriores.

El RCV fue estimado a partir de la ecuación descrita por D’Agostino y cols. ${ }^{(29)}$, teniendo en cuenta las variables: edad, concentraciones séricas de colesterol total y colesterol HDL, antecedentes de tratamiento antihipertensivo, consumo de tabaco y DM2, a través de lo cual fue calculado el porcentaje de riesgo de sufrir un evento cardiovascular en 10 años.

Para la genotipificación, se escogieron seis SNPs, usando los datos de la población CEU del proyecto HapMap, tomando como referencia aquellos polimorfismos con una frecuencia alélica menor $(F A M) \geq 0,25$, y un coeficiente de correlación $\left(r^{2}\right) \geq 0,8$. De acuerdo con esto los SNPs analizados fueron: (i) rs926198, (ii) rs3779512, (iii) rs10270569, (iv) rs11773845, (v) rs7804372 y (vi) rs1049337. Estas variantes fueron genotipificadas por reacción en cadena de la polimerasa cuantitativa (qPCR, del inglés quantitative Polymerase Chain Reaction) usando sondas TaqMan específicas (Thermo Fisher Scientific, Inc., Waltham, MA, Estados Unidos). La discriminación alélica se llevó a cabo con los datos de fluorescencia, mediante el StepOne Real-Time PCR Software (Thermo Fisher Scientific, Inc., Waltham, MA, Estados Unidos).

En los procedimientos estadísticos, se emplearon medidas de tendencia central para describir los datos antropométricos y de bioquímica sanguínea, así como el puntaje de RCV; por su parte, las alteraciones metabólicas fueron descritas con medidas de frecuencia. Las medias fueron comparadas con la prueba T de Student, mientras que las frecuencias fueron comparadas con la prueba exacta de Fisher o de $\mathrm{X}^{2}$, según fuera apropiado. 
Las frecuencias alélicas y genotípicas fueron determinadas empleando el paquete estadístico genetics $^{(30)}$. Las asociaciones entre las variables continuas de acuerdo con la distribución genotípica fueron comparadas mediante un análisis de varianza (ANOVA). Para la comparación de promedios de variables continuas entre los grupos de genotipos de los SNPs se aplicó la prueba Diferencia Honestamente Significativa de Tukey o Tukey's HSD (del inglés, Honestly Significantly Diferent). Adicionalmente, los datos fueron analizados mediante un modelo de regresión generalizada (regresión lineal multivariada) y ajustados por la corrección de Bonferroni, para lo cual se consideraron como significativos valores de $p<0,0083$. Además, se construyeron árboles de regresión que describieron las relaciones entre variables respuestas (puntaje de RCV) y las variables explicativas o predictoras (SNPs). Este análisis se realizó mediante el paquete rpart $^{(31)}$. Todos los procedimientos antes mencionados fueron realizados mediante el programa estadístico $\mathrm{R}$ versión 3.3.2 ${ }^{(32)}$.

\section{Resultados}

\section{Características demográficas}

La muestra estuvo comprendida por 645 sujetos, de los cuales 50 fueron excluidos. Se analizaron los datos de 595 individuos $(59,7 \%$ hombres y $40,3 \%$ mujeres). La mayor parte de los sujetos $(77,5 \%)$ habitaban en estratos económicos 1 y 2 , el $17,6 \%$ en 3 , y los restantes en estratos 4,5 y 6 . El promedio de edad en la población fue de $43,7 \pm 17,4$ años (tabla 1).

\section{Datos bioquímicos, antecedentes (HTA, tabaco y DM2) y RCV}

La media de los niveles séricos de colesterol total y HDL en la población general fue de $188,5 \pm 55,9$ y 46,1 $\pm 15,1$, respectivamente, siendo superior en el grupo de mujeres que en el de hombres, para ambas variables; la media de la tensión arterial sistólica fue de 113,9 $\pm 17,0 \mathrm{mmHg}$ y la diastólica de 75,5 $\pm 10,7$ mmHg. La HTA fue el antecedente que se presentó con mayor frecuencia $(n=143,24 \%)$, seguido por el consumo de tabaco $(n=121,20,3 \%)$ y la DM2 (n= 78, 13,1\%) en la población general. La frecuencia de antecedentes de HTA y diabetes fue superior en el grupo de mujeres, mientras que la frecuencia de antecedente de tabaco fue mayor en los hombres. El promedio de puntaje de RCV fue de $8,9 \pm 13,2$ (tabla 1).

\section{Genotipificación}

En los 595 individuos incluidos se obtuvieron los genotipos de los 6 polimorfismos seleccionados en el gen CAV1:

- rs3779512, FAM (alelo C) de 30,5\%

- rs926198, FAM (alelo T) de 40,1\%

- rs10270569, FAM (alelo C) de 19,3\%

- rs11773845, FAM (alelo C) de 43,4\%

- rs7804372, FAM (alelo A) de 26,6\%

- rs1049337, FAM (alelo T) de 35,6\%

Las frecuencias alélicas y distribuciones genotípicas se muestran en la tabla 2.

Tabla 1. Descripción de variables en la población general

\begin{tabular}{|c|c|c|c|c|c|c|c|}
\hline \multirow{2}{*}{$\begin{array}{l}\text { Variables } \\
\text { Edad }\end{array}$} & \multicolumn{2}{|c|}{$\begin{array}{l}\text { Hombres } \\
(\mathrm{n}=355)\end{array}$} & \multicolumn{2}{|c|}{$\begin{array}{l}\text { Mujeres } \\
(n=240)\end{array}$} & \multicolumn{2}{|c|}{ Población general } & \multirow{2}{*}{$\begin{array}{l}\text { Valores de } \mathbf{p} \\
7,319 \times 10^{-9}\end{array}$} \\
\hline & 40,2 & $\pm 14,5$ & 48,8 & $\pm 18,6$ & 43,7 & $\pm 17,4$ & \\
\hline \multicolumn{8}{|c|}{ Variables bioquímicas } \\
\hline $\begin{array}{l}\text { Colesterol } \\
\text { total }\end{array}$ & 183,6 & $\pm 52,6$ & 195,8 & $\pm 59,9$ & 188,5 & $\pm 55,9$ & 0,011 \\
\hline $\begin{array}{l}\text { Colesterol } \\
\text { HDL }\end{array}$ & 44,1 & $\pm 12,3$ & 49,2 & $\pm 18,1$ & 46,1 & $\pm 15,1$ & 0,0001 \\
\hline \multicolumn{8}{|c|}{ Presión arterial } \\
\hline TAS & 113,7 & $\pm 15,2$ & 114,4 & $\pm 19,5$ & 113,9 & $\pm 17,0$ & 0,608 \\
\hline TAD & 76,6 & $\pm 10,3$ & 73,9 & $\pm 11,3$ & 75,5 & $\pm 10,7$ & 0,004 \\
\hline \multicolumn{8}{|c|}{ Antecedentes } \\
\hline A. HTA & 56 & $(15,8)$ & 87 & $(36,2)$ & 143 & $(24,0)$ & $1,736 \times 10^{-8}$ \\
\hline A. Cigarrillo & 86 & $(24,2)$ & 35 & $(14,6)$ & 121 & $(20,3)$ & 0,005 \\
\hline A. DM & 24 & $(6,7)$ & 54 & $(22,5)$ & 78 & $(13,1)$ & $4,85 \times 10^{-8}$ \\
\hline RCV & 8,8 & $\pm 14,3$ & 8,9 & $\pm 11,4$ & 8,7 & $\pm 13,2$ & 0,914 \\
\hline
\end{tabular}


Tabla 2. Frecuencias alélicas y distribución para el gen $C A V 1$

\begin{tabular}{|c|c|c|c|c|}
\hline \multicolumn{3}{|c|}{ CAV1 } & \multirow[t]{2}{*}{$F_{\text {is }}$} & \multirow[t]{2}{*}{ Valor de $p$} \\
\hline rs3779512 & \multicolumn{2}{|c|}{ n (\%) } & & \\
\hline G & 713 & $(59,9)$ & \multirow{5}{*}{0,05} & \multirow{5}{*}{0,09} \\
\hline $\mathrm{T}$ & 477 & $(40,1)$ & & \\
\hline GG & 221 & $(37,1)$ & & \\
\hline GT & 271 & $(45,6)$ & & \\
\hline TT & 103 & $(17,3)$ & & \\
\hline rs926198 & \multicolumn{2}{|c|}{$n(\%)$} & & \\
\hline C & 363 & $(30,5)$ & \multirow{5}{*}{0,03} & \multirow{5}{*}{0,19} \\
\hline $\mathrm{T}$ & 827 & $(69,5)$ & & \\
\hline CC & 61 & $(10,2)$ & & \\
\hline CT & 241 & $(40,5)$ & & \\
\hline TT & 293 & $(49,3)$ & & \\
\hline rs10207569 & \multicolumn{2}{|c|}{ n (\%) } & & \\
\hline C & 961 & $(19,3)$ & \multirow{5}{*}{0,004} & \multirow{5}{*}{0,50} \\
\hline T & 229 & $(80,7)$ & & \\
\hline CC & 388 & $(65,2)$ & & \\
\hline CT & 185 & $(31,1)$ & & \\
\hline TT & 22 & $(3,7)$ & & \\
\hline rs11773845 & \multicolumn{2}{|c|}{ n (\%) } & & \\
\hline A & 674 & $(56,6)$ & \multirow{5}{*}{0,07} & \multirow{5}{*}{0,04} \\
\hline C & 516 & $(43,4)$ & & \\
\hline $\mathrm{AA}$ & 201 & $(33,8)$ & & \\
\hline AC & 272 & $(45,7)$ & & \\
\hline CC & 122 & $(20,5)$ & & \\
\hline rs7804372 & \multicolumn{2}{|c|}{ n (\%) } & & \\
\hline T & 873 & $(73,4)$ & \multirow{5}{*}{0,03} & \multirow{5}{*}{0,24} \\
\hline A & 317 & $(26,6)$ & & \\
\hline TT & 324 & $(54,5)$ & & \\
\hline TA & 225 & $(37,8)$ & & \\
\hline AA & 46 & $(7,7)$ & & \\
\hline rs1049737 & \multicolumn{2}{|c|}{ n (\%) } & & \\
\hline C & 814 & $(64,4)$ & \multirow{5}{*}{0,06} & \multirow{5}{*}{0,07} \\
\hline T & 376 & $(35,6)$ & & \\
\hline CC & 298 & $(50,1)$ & & \\
\hline CT & 218 & $(36,6)$ & & \\
\hline TT & 79 & $(13,3)$ & & \\
\hline
\end{tabular}

\section{Frecuencias genotípicas y asociaciones con RCV}

Los modelos de regresión lineal simple en los que se consideró como variable explicativa cada uno de los SNPs (por separado) y como variable respuesta los valores de RCV, mostraron significancia estadística solo para la variante rs1049337 ( $p=0,0001$ ) (tabla 3). Tras realizar la prueba Tukey's HSD, el genotipo TT del SNP rs1049337 mostró mayores puntajes de RCV que los otros genotipos (CC у TT) ( $p=0,02), 4,17 \%$ [IC95\% $0,26-8,08 \%$ ] más que CC $(p=0,03)$ y $4,36 \%$ [IC95\% $0,3-8,4 \%$ ] más que CT $(p=0,03)$.

El modelo de regresión lineal multivariada (que incluyó como variables explicativas todos los SNPs y como variable dependiente el puntaje de RCV) también mostró diferencias significativas para el SNP rs1049337 $(p=0,0001)$, considerándose como significativos valores de $p<0,0083$ (corrección por Bonferroni).

El árbol de regresión siguió la misma tendencia, pero además mostró que los sujetos con este genotipo (TT para el SNP rs1049337) mostraban un mayor puntaje de riesgo si además contaban con el genotipo TT para el SNP rs3779512 $(p=0,0005)$ (figura $\mathbf{1}$ ).

\section{Discusión}

La caveolina 1 es una proteína integral de membrana que ha sido relacionada con alteraciones metabólicas como hipertensión, resistencia a la insulina, DM2 y anormalidades lipídicas que incrementan el riesgo de padecer enfermedades de tipo cardiovascular ${ }^{(19,33,34)}$. El análisis de variantes polimórficas presentes en el gen que la codifica ha venido siendo objeto de estudio, con el fin de comprender con mayor profundidad su papel en el desarrollo y progresión de este tipo de patologías. En el presente trabajo se analizó la asociación entre variantes polimórficas en el gen CAV1 y los puntajes de RCV en una población genéticamente heterogénea de adultos del caribe colombiano. Los resultados mostraron que las variantes rs1049337 y rs3779512 se encontraron asociadas a incrementos en el puntaje de riesgo.

De los seis SNP seleccionados, solo el SNP rs1049337 se encontró asociado de manera significativa con el RCV, particularmente el genotipo TT que mostró mayores valores del puntaje que el resto de los genotipos. Adicionalmente, el análisis mediante los árboles de regresión en este mismo grupo (TT para rs1049337) mostró que el SNP rs3779512 (específicamente el genotipo TT) se encontraba asociado a mayores valores del puntaje. Sobre este hallazgo, no se reportan estudios que asocien estas dos variantes con alteraciones cardiometabólicas. El SNP rs1049337 se ha vinculado a asociaciones con el riesgo de carcinoma renal y cáncer de próstata ${ }^{(35,36)}$, así como con el incremento del riesgo de patologías oculares, principalmente glaucoma de ángulo abierto, esta última condición también relacionada con el SNP rs3779512, sin encontrar 
Tabla 3. Valores promedio de riesgo cardiovascular (RCV) de acuerdo con los genotipos de los SNP

\begin{tabular}{|c|c|c|c|c|c|c|}
\hline SNP & \multicolumn{3}{|c|}{ Genotipos $^{+}$} & \multicolumn{3}{|c|}{ Valor de p } \\
\hline \multirow{2}{*}{ rs3779512 } & GG & GT & TT & & GG vs GT+TT & TT vs GT+GG \\
\hline & $8,7 \pm 14,13$ & $8,65 \pm 12,13$ & $9,7 \pm 13,9$ & 0,515 & 0,736 & 0,25 \\
\hline \multirow{2}{*}{ rs926198 } & $\mathrm{CC}$ & $\mathrm{CT}$ & TT & & CC vs $\mathrm{CT}+\mathrm{TT}$ & TT vs $C T+C C$ \\
\hline & $7,8 \pm 13,64$ & $9,2 \pm 13,11$ & $8,7 \pm 13,24$ & 0,455 & 0,32 & 0,695 \\
\hline \multirow{2}{*}{ rs10207569 } & $\mathrm{CC}$ & $\mathrm{CT}$ & TT & & CC vs $\mathrm{CT}+\mathrm{TT}$ & TT vs $C T+C C$ \\
\hline & $8,65 \pm 13,5$ & $9,18 \pm 12,47$ & $9,82 \pm 13,27$ & 0,672 & 0,408 & 0,586 \\
\hline \multirow{2}{*}{ rs11773845 } & AA & $A C$ & $\mathrm{CC}$ & & $A A$ vs $A C+C C$ & $C C$ vs $A C+A A$ \\
\hline & $9,4 \pm 14,8$ & $8,2 \pm 10,9$ & $9,32 \pm 14,93$ & 0,267 & 0,226 & 0,491 \\
\hline \multirow{2}{*}{ rs7804372 } & TT & $\mathrm{TA}$ & AA & & TT vs $T A+A A$ & $A A$ vs $T A+T T$ \\
\hline & $9,0 \pm 14,2$ & $8,4 \pm 11,59$ & $9,98 \pm 13,65$ & 0,472 & 0,65 & 0,349 \\
\hline \multirow{2}{*}{ rs1049737 } & $\mathrm{CC}$ & $\mathrm{CT}$ & TT & & CC vs CT+TT & TT vs $C T+C C$ \\
\hline & $8,37 \pm 11,3$ & $8,18 \pm 12,31$ & $12,54 \pm 20,13$ & $0,0001^{*}$ & 0,16 & $2,88 \times 10-5$ \\
\hline
\end{tabular}

+Promedio \pm Desviación estándar.

*valores de $p<0,05$. Los datos fueron corregidos por sexo y edad.

Figura 1. Árbol de regresión que esquematiza las asociaciones entre el puntaje de riesgo cardiovascular (RCV) y los genotipos de los SNPs analizados: rs3779512, rs926198, rs10207569, rs11773845, rs7804372, rs1049737. El genotipo TT para el SNP rs1049337 mostró un mayor puntaje de RCV en comparación con los genotipos CC o CT $\left(p=2,88 \times 10^{-5}\right)$, a su vez, el genotipo TT para el SNP rs3779512

incrementó aún más el valor $(p=0,0002)$. En este esquema se encuentran representadas solo las variables que tuvieron influencia sobre el desenlace. Los valores fueron ajustados por edad y sexo.

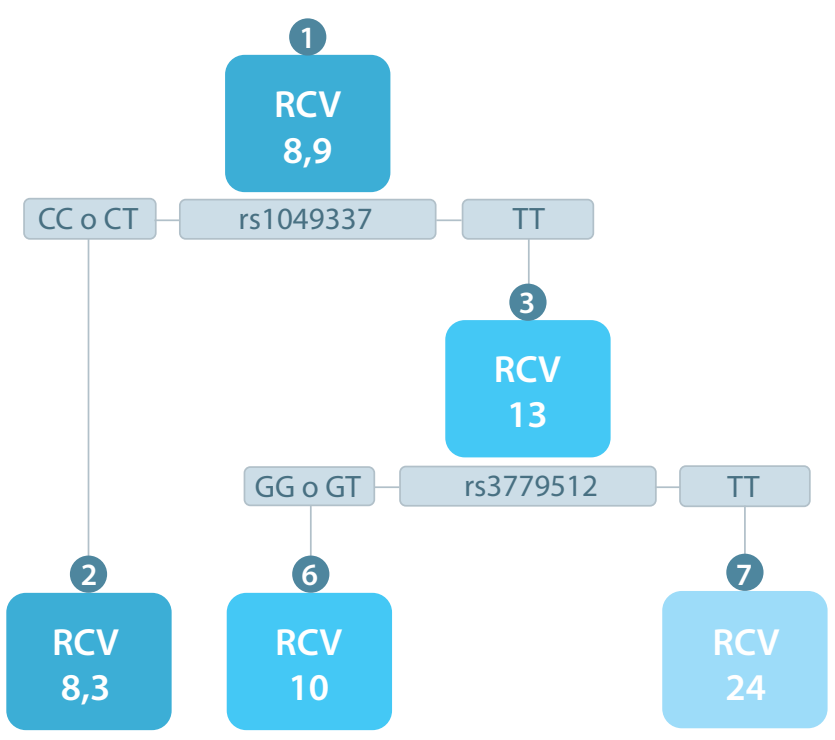

diferencias significativas ${ }^{(37,38)}$ y con el SNP rs10270569, para el cual sí se describen asociaciones que conservan significancia estadística $^{(39)}$. Aunque no se evidencian estudios que relacionen estas dos variantes (rs1049337 y rs3779512) a patologías de tipo cardiovascular, es posible que las asociaciones encontradas en el presente trabajo estén relacionadas con cambios en la expresión del gen $C A V 1$, y así mismo de la proteína caveolina 1 , proteína implicada en la homeostasis lipídica, regulación de procesos metabólicos y recientemente relacionada con la fisiopatología de trastornos hipertensivos ${ }^{(40)}$.

De acuerdo con los resultados del presente trabajo, el gen CAV1 no se encontró asociado a DM2, contrario a lo encontrado por otros autores en poblaciones con ancestros europeos y en grupos hispanos residentes de Estados Unidos, donde el SNP rs926198 estuvo asociado con resistencia a la insulina y DM2 $2^{(19,20)}$. Sobre este punto, vale la pena resaltar que el mencionado trabajo realizado por Baudrand y cols. (2015), tuvo en cuenta consideraciones más estrictas para el diagnóstico de estas condiciones, incluyendo además la resistencia a la insulina como una de sus variables. En el presente trabajo por otro lado, dichas asociaciones fueron analizadas a partir del hallazgo de DM2, establecido bien sea por valores de glicemia en ayuno $\geq 126 \mathrm{mg} / \mathrm{dL}$ o por antecedentes personales referidos por el sujeto bajo estudio. Roberts y cols. (2009) reportaron asociaciones significativas con menores riesgos de hipertensión portopulmonar ${ }^{(41)}$. En el presente trabajo esta variante se encontró asociada a los valores de tensión arterial tanto sistólica como diastólica $(p<0,05)$ [no se muestra en el manuscrito]. En otros estudios se 
ha analizado la relación de dicha variante con el riesgo de patologías como esclerosis sistémica y cáncer de próstata, sin encontrar asociaciones ${ }^{(36,42)}$.

En el presente trabajo no se reportaron asociaciones para los SNP rs11773845 y rs7804372 con ninguna de las condiciones estudiadas. Esto coincide con los hallazgos de Chen y cols. (2016), en los que no encontró asociación del SNP rs11773845 con enfermedad arterial coronaria ${ }^{(43)}$. Sin embargo, existe evidencia que relaciona a estas dos variantes con alteraciones cardiacas y metabólicas; Pojoga y cols. (2011) reportaron asociación del SNP rs3807989 (proxy para rs11773845) con resistencia a la insulina (20) y Liu y cols. (2017) recientemente reportaron asociaciones de este polimorfismo con fibrilación auricular ${ }^{(33)}$. En el presente trabajo no se estudiaron de forma específica las condiciones valoradas por otros autores (i.e., resistencia a la insulina y fibrilación auricular), pero por otro lado el objeto de estudio aquí planteado guarda más relación con lo estudiado por Chen y cols. (2016) (i.e., enfermedad arterial coronaria), que reportó resultados que conservan el sentido de los hallazgos del presente trabajo. De acuerdo con esto, este SNP podría entonces estar involucrado en el desarrollo de patologías cardiometabólicas, pero participando en las vías bioquímicas del metabolismo de carbohidratos o en la conducción de señales.

Con respecto al SNP rs7804372, recientemente Shyu y cols. (2017) reportaron asociaciones del alelo A de esta variante con incrementos en el riesgo de accidente cerebrovas- cular por enfermedad aterosclerótica en grandes vasos (OR 1,63 IC 1,19-2,22) ${ }^{(44)}$. Así mismo, el polimorfismo rs3807994 (proxy de esta variante rs7804372) se ha asociado a mayores niveles séricos de caveolina $1^{(45)}$, considerándose a esta última como un determinante crítico en la autofagia, recambio metabólico, estrés oxidativo del endotelio vascular, y la señalización involucrada en el desarrollo de patologías cardiovasculares $^{(46,47)}$.

A pesar de las asociaciones estadísticas encontradas, este estudio presenta varias limitaciones que deben considerarse. Tratándose de una población de estudio mezclada se debió considerar la realización de una corrección de los datos, teniendo en cuenta el ancestro. Este trabajo cuenta con un bajo poder de estudio, por lo que variables confundidoras pudieron influir en los resultados, por ello se llevaron a cabo rigurosamente los ajustes por sexo y edad con el objeto de reducir dichos sesgos. Es necesario realizar estudios con mayor profundidad para el establecimiento de asociaciones significativas en relación a este parámetro (riesgo cardiovascular).

\section{Agradecimientos}

Este estudio fue posible gracias a la ayuda y apoyo del grupo de investigación UNIMOL y al grupo interdisciplinar de profesionales de la facultad de Medicina de la Universidad de Cartagena. 


\section{Referencias}

1. World Health Organization. Global Atlas on Cardiovascular Disease Prevention and Control: World Health Organization; 2013.

2. Goff DC, Jr., Lloyd-Jones DM, Bennett G, Coady S, D’Agostino RB, Gibbons R, et al. 2013 ACC/AHA guideline on the assessment of cardiovascular risk: a report of the American College of Cardiology/American Heart Association Task Force on Practice Guidelines. Circulation. 2014;129(25 Suppl 2):S49-73.

3. Piepoli MF, Hoes AW, Agewall S, Albus C, Brotons C, Catapano AL, et al. 2016 European Guidelines on cardiovascular disease prevention in clinical practice: The Sixth Joint Task Force of the European Society of Cardiology and Other Societies on Cardiovascular Disease Prevention in Clinical Practice (constituted by representatives of 10 societies and by invited experts)Developed with the special contribution of the European Association for Cardiovascular Prevention \& Rehabilitation (EACPR). European heart journal. 2016;37(29):2315-81.

4. Fernando L, Pamela S, Alejandra L. Cardiovascular disease in Latin America: the growing epidemic. Progress in cardiovascular diseases. 2014;57(3):262-7.

5. World Health Organization. Global status report on noncommunicable diseases 2014. Geneva: World Health Organization; 2014. 280 p. p.

6. Amouyel P, Deverly A. [Global cardiovascular risk: definition, evaluation and management strategies. Round table no. 1. XV]. Therapie. 2000;55(4):533-9.

7. deGoma EM, Dunbar RL, Jacoby D, French B. Differences in absolute risk of cardiovascular events using risk-refinement tests: a systematic analysis of four cardiovascular risk equations. Atherosclerosis. 2013:227(1):172-7.

8. Sotos-Prieto M, Baylin A, Campos H, Qi L, Mattei J. Lifestyle Cardiovascular Risk Score, Genetic Risk Score, and Myocardial Infarction in Hispanic/Latino Adults Living in Costa Rica. Journal of the American Heart Association. 2016;5(12).

9. Humphries SE, Cooper JA, Talmud PJ, Miller GJ. Candidate gene genotypes, along with conventional risk factor assessment, improve estimation of coronary heart disease risk in healthy UK men. Clinical chemistry. 2007;53(1):8-16.

10. Morrison AC, Bare LA, Chambless LE, Ellis SG, Malloy M, Kane JP, et al. Prediction of coronary heart disease risk using a genetic risk score: the Atherosclerosis Risk in Communities Study. American journal of epidemiology. 2007;166(1):28-35.

11. Bolton JL, Stewart MC, Wilson JF, Anderson N, Price JF. Improvement in prediction of coronary heart disease risk over conventional risk factors using SNPs identified in genome-wide association studies. PloS one. 2013;8(2):e57310.

12. Beaney KE, Cooper JA, Ullah Shahid S, Ahmed W, Qamar R, Drenos F, et al. Clinical Utility of a Coronary Heart Disease Risk Prediction Gene Score in UK Healthy Middle Aged Men and in the Pakistani Population. PloS one. 2015;10(7):e0130754.

13. Companioni O, Rodriguez Esparragon F, Fernandez-Aceituno AM, Rodriguez Perez JC. [Genetic variants, cardiovascular risk and genome-wide association studies]. Revista espanola de cardiologia. 2011;64(6):509-14.

14. Mora Garcia G, Gomez Alegria C, Gomez Camargo D. Caveolin 1 (CAV1) gene polymorphisms associated to body mass index, hypertension and obesity related disorders in adults from Caribbean population. Obesity Reviews. 2016;17(Suppl. 2):5 - 20.

15. Engelman JA, Zhang XL, Lisanti MP. Sequence and detailed organization of the human caveolin-1 and -2 genes located near the D7S522 locus (7q31.1). Methylation of a CpG island in the 5' promoter region of the caveolin-1 gene in human breast cancer cell lines. FEBS letters. 1999;448(2-3):221-30.

16. Kurzchalia TV, Dupree P, Monier S. VIP21-Caveolin, a protein of the transGolgi network and caveolae. FEBS letters. 1994;346(1):88-91.

17. Yamada E. The fine structure of the gall bladder epithelium of the mouse. The Journal of biophysical and biochemical cytology. 1955;1(5):445-58

18. Liu GZ, Liang B, Lau WB, Wang Y, Zhao J, Li R, et al. High glucose/High Lipids impair vascular adiponectin function via inhibition of caveolin-1/AdipoR1 signalsome formation. Free radical biology \& medicine. 2015;89:473-85.

19. Baudrand R, Goodarzi MO, Vaidya A, Underwood PC, Williams JS, Jeunemaitre $\mathrm{X}$, et al. A prevalent caveolin-1 gene variant is associated with the metabolic syndrome in Caucasians and Hispanics. Metabolism: clinical and experimental. 2015;64(12):1674-81.

20. Pojoga LH, Underwood PC, Goodarzi MO, Williams JS, Adler GK, Jeunemaitre $\mathrm{X}$, et al. Variants of the caveolin-1 gene: a translational investigation linking insulin resistance and hypertension. The Journal of clinical endocrinology and metabolism. 2011;96(8):E1288-92.

21. Grilo A, Fernandez ML, Beltran M, Ramirez-Lorca R, Gonzalez MA, Royo JL, et al. Genetic analysis of CAV1 gene in hypertension and metabolic syndrome. Thrombosis and haemostasis. 2006;95(4):696-701.

22. Razani B, Combs TP, Wang XB, Frank PG, Park DS, Russell RG, et al. Caveolin1-deficient mice are lean, resistant to diet-induced obesity, and show hypertriglyceridemia with adipocyte abnormalities. The Journal of biological chemistry. 2002;277(10):8635-47.
23. Gomez Camargo D, Camacho-Mejorado R, Gomez Alegria C, Alario Bello A, Hernandez-Tobias EA, Mora Garcia G, et al. Genetic structure of Cartagena de Indias population using hypervariable markers of $\mathrm{Y}$ chromosome. Open Journal of Genetics. 2015;5(1):1-20.

24. Builes JJ, Martinez B, Gomez A, Caraballo L, Espinal C, Aguirre D, et al. Y chromosome STR haplotypes in the Caribbean city of Cartagena (Colombia). Forensic science international. 2007;167(1):62-9.

25. Noguera MC, Schwegler A, Gomes V, Briceno I, Alvarez L, Uricoechea D, et al. Colombia's racial crucible: Y chromosome evidence from six admixed communities in the Department of Bolivar. Ann Hum Biol. 2014;41(5):453-9.

26. Vergara C, Murray T, Rafaels N, Lewis R, Campbell M, Foster C, et al. African ancestry is a risk factor for asthma and high total IgE levels in African admixed populations. Genetic epidemiology. 2013;37(4):393-401.

27. Alberti KG, Zimmet P, Shaw J. The metabolic syndrome--a new worldwide definition. Lancet. 2005;366(9491):1059-62.

28. James PA, Oparil S, Carter BL, Cushman WC, Dennison-Himmelfarb C, Handler J, et al. 2014 evidence-based guideline for the management of high blood pressure in adults: report from the panel members appointed to the Eighth Joint National Committee (JNC 8). JAMA : the journal of the American Medical Association. 2014;311(5):507-20.

29. D’Agostino RB, Sr., Vasan RS, Pencina MJ, Wolf PA, Cobain M, Massaro JM, et al. General cardiovascular risk profile for use in primary care: the Framingham Heart Study. Circulation. 2008;117(6):743-53.

30. Gregory Warnes and with contributions from Gregor Gorjanc and Friedrich Leisch and Michael Man. (2013). genetics: Population Genetics. R package version 1.3.8.1. https://CRAN.R-project.org/package=genetics.

31. Terry Therneau BAaBR. rpart: Recursive Partitioning and Regression Trees. $\mathrm{R}$ package version 4.1-10. https://CRAN.R-project.org/package=rpart.

32. R Core Team (2016). R: A language and environment for statistical computing. R Foundation for Statistical Computing, Vienna, Austria. URL http:// www.R-project.org/.

33. Liu L, Ebana Y, Nitta JI, Takahashi Y, Miyazaki S, Tanaka T, et al. Genetic Variants Associated With Susceptibility to Atrial Fibrillation in a Japanese Population. Can J Cardiol. 2017;33(4):443-9.

34. Asker S, Taspinar M, Koyun H, Ozbay B, Arisoy A. Caveolin-1 polymorphisms in patients with severe obstructive sleep apnea. Biomarkers. 2017;22(1):77-80.

35. Zhao R, Liu K, Huang Z, Wang J, Pan Y, Huang Y, et al. Genetic Variants in Caveolin-1 and RhoA/ROCK1 Are Associated with Clear Cell Renal Cell Carcinoma Risk in a Chinese Population. PloS one. 2015;10(6):e0128771.

36. Langeberg WJ, Tahir SA, Feng Z, Kwon EM, Ostrander EA, Thompson TC et al. Association of caveolin-1 and -2 genetic variants and post-treatment serum caveolin-1 with prostate cancer risk and outcomes. Prostate. 2010;70(9):1020-35.

37. Liu Y, Hauser MA, Akafo SK, Qin X, Miura S, Gibson JR, et al. Investigation of known genetic risk factors for primary open angle glaucoma in two populations of African ancestry. Invest Ophthalmol Vis Sci. 2013;54(9):6248-54.

38. Rong SS, Chen LJ, Leung CK, Matsushita K, Jia L, Miki A, et al. Ethnic specific association of the CAV1/CAV2 locus with primary open-angle glaucoma. Sci Rep. 2016;6:27837.

39. Wiggs JL, Kang JH, Yaspan BL, Mirel DB, Laurie C, Crenshaw A, et al. Common variants near CAV1 and CAV2 are associated with primary open-angle glaucoma in Caucasians from the USA. Hum Mol Genet. 2011;20(23):4707-13.

40. Chen YH, Lin WW, Liu CS, Hsu LS, Lin YM, Su SL. Caveolin-1 Expression Ameliorates Nephrotic Damage in a Rabbit Model of Cholesterol-Induced Hypercholesterolemia. PloS one. 2016;11(4):e0154210.

41. Roberts KE, Fallon MB, Krowka MJ, Brown RS, Trotter JF, Peter I, et al. Genetic risk factors for portopulmonary hypertension in patients with advanced liver disease. Am J Respir Crit Care Med. 2009;179(9):835-42.

42. Manetti M, Allanore Y, Saad M, Fatini C, Cohignac V, Guiducci S, et al. Evidence for caveolin-1 as a new susceptibility gene regulating tissue fibrosis in systemic sclerosis. Ann Rheum Dis. 2012;71(6):1034-41.

43. Chen S, Wang X, Wang J, Zhao Y, Wang D, Tan C, et al. Genomic variant in CAV1 increases susceptibility to coronary artery disease and myocardial infarction. Atherosclerosis. 2016;246:148-56.

44. Shyu HY, Chen MH, Hsieh YH, Shieh JC, Yen LR, Wang HW, et al. Association of eNOS and Cav-1 gene polymorphisms with susceptibility risk of large artery atherosclerotic stroke. PloS one. 2017;12(3):e0174110.

45. Kastelijn EA, van Moorsel CH, Kazemier KM, Roothaan SM, Ruven HJ, Kwakkel-van Erp JM, et al. A genetic polymorphism in the CAV1 gene associates with the development of bronchiolitis obliterans syndrome after lung transplantation. Fibrogenesis Tissue Repair. 2011;4:24.

46. Shiroto T, Romero N, Sugiyama T, Sartoretto JL, Kalwa H, Yan Z, et al. Caveolin-1 is a critical determinant of autophagy, metabolic switching, and oxidative stress in vascular endothelium. PloS one. 2014;9(2):e87871.

47. Williams JJ, Palmer TM. Cavin-1: caveolae-dependent signalling and cardiovascular disease. Biochem Soc Trans. 2014;42(2):284-8. 